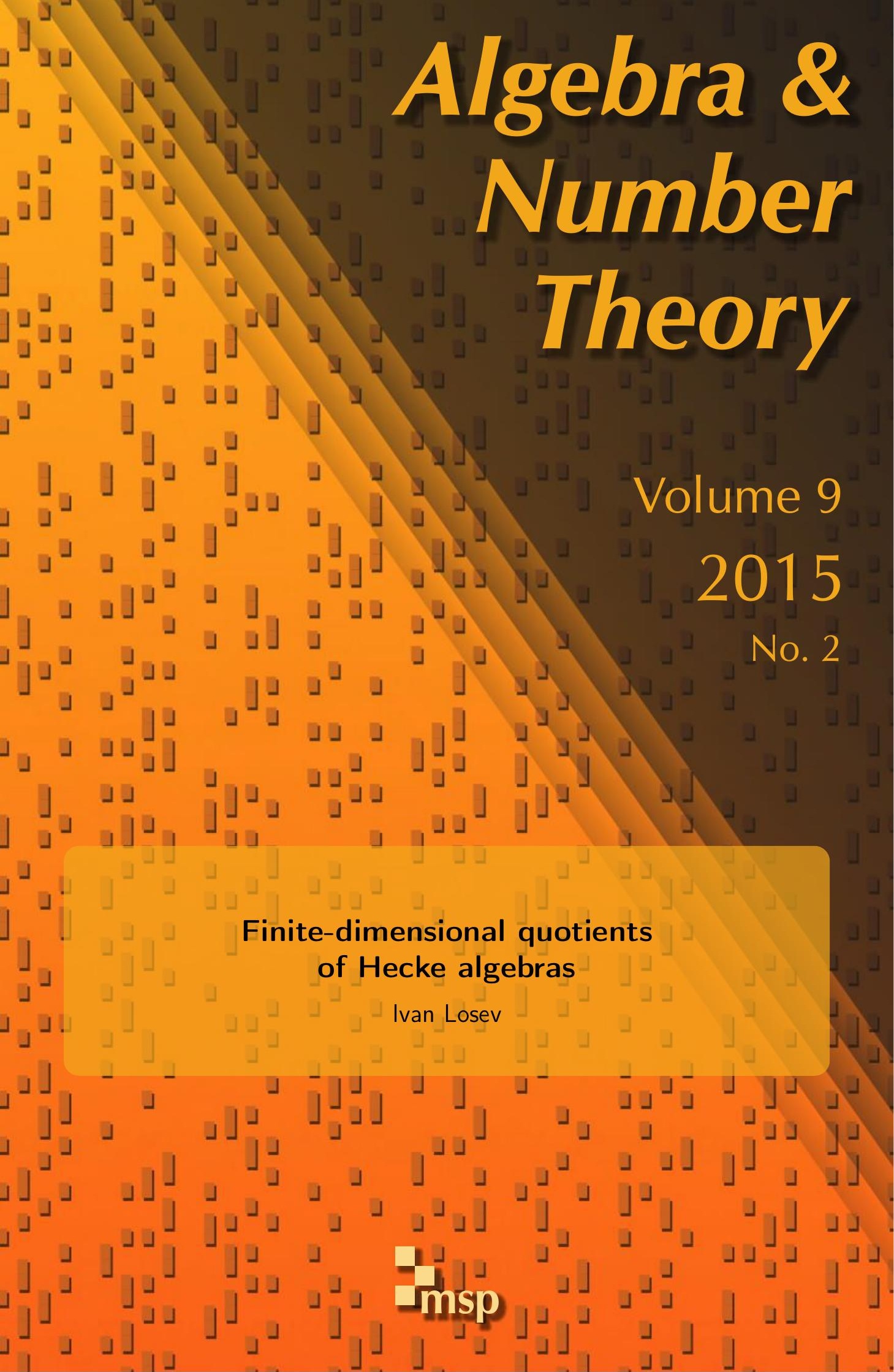




\title{
Finite-dimensional quotients of Hecke algebras
}

\author{
Ivan Losev
}

Let $W$ be a complex reflection group. We prove that there is a maximal finitedimensional quotient of the Hecke algebra $\mathscr{H}_{q}(W)$ of $W$, and that the dimension of this quotient coincides with $|W|$. This is a weak version of a 1998 BrouéMalle-Rouquier conjecture. The proof is based on the categories 0 for rational Cherednik algebras.

\section{Introduction}

Let $W$ be a complex reflection group. Recall that such groups are fully classified; see [Shephard and Todd 1954]. In this context, one can also define the braid group $B_{W}$. Namely, let $\mathfrak{h}$ denote the reflection representation of $W$. Inside $\mathfrak{h}$, one considers the open subset of regular points $\mathfrak{h}^{\text {reg }}:=\left\{x \in \mathfrak{h} \mid W_{x}=\{1\}\right\}$, so that $W$ is the Galois group of the cover $\mathfrak{h}^{\text {reg }} \rightarrow \mathfrak{h}^{\text {reg }} / W$. By definition, the braid group $B_{W}$ is the fundamental group $\pi_{1}\left(\mathfrak{h}^{\text {reg }} / W\right)$.

If $W$ is a Coxeter group, one considers a flat deformation of $\mathbb{C} W$, called a Hecke algebra. These algebras are of importance in representation theory (e.g., that of finite groups of Lie type) and beyond (e.g., in knot theory). One can define Hecke algebras for complex reflection groups as well. In the general case, this was done in [Broué et al. 1998, Section 4C]. To recall the definition, we need some more notation. Namely, let $\mathfrak{H}$ denote the set of reflection hyperplanes for $W$. For $\Gamma \in \mathfrak{H}$, let $W_{\Gamma}$ denote the pointwise stabilizer of $\Gamma$; this is a cyclic group. Set $\ell_{\Gamma}:=\left|W_{\Gamma}\right|$. The group $B_{W}$ is generated by elements $T_{\Gamma}, \Gamma \in \mathfrak{H}$, where, roughly speaking, $T_{\Gamma}$ is the rotation around $\Gamma$ by $2 \pi / \ell_{\Gamma}$; see [loc. cit., Section 2]. Now, pick independent variables $u_{\Gamma, i}, i=0,1, \ldots, \ell_{\Gamma}-1$ with $u_{\Gamma, i}=u_{\Gamma^{\prime}, i}$ for $W$-conjugate $\Gamma, \Gamma^{\prime}$. Set $\boldsymbol{u}:=\left(u_{\Gamma, i}\right)$. By definition ([loc. cit., Definition 4.21]), the Hecke algebra $\mathscr{H}_{\boldsymbol{u}}(W)$ is the quotient of $\mathbb{Z}\left[\boldsymbol{u}^{ \pm 1}\right] B_{W}$ by the relations

$$
\prod_{i=0}^{\ell_{\Gamma}^{-1}}\left(T_{\Gamma}-u_{\Gamma, i}\right)=0 .
$$

MSC2010: primary 20C08; secondary 20F55, 16G99.

Keywords: Hecke algebras, rational Cherednik algebras, categories $\mathrm{O}, \mathrm{KZ}$ functor. 
Broué, Malle and Rouquier [Broué et al. 1998, Section 4C] conjectured that $\mathscr{H}_{\boldsymbol{u}}(W)$ is a free $\mathbb{Z}\left[\boldsymbol{u}^{ \pm 1}\right]$-module generated by $|W|$ elements. Currently, the proof is missing in the case of several exceptional complex reflection groups. In this paper, we are going to prove a weaker version of this conjecture.

First of all, we are dealing with specializations to $\mathbb{C}$. For a collection of nonzero complex numbers $\left(q_{\Gamma, i}\right)$, where $\Gamma \in \mathfrak{H} / W$ and $i \in\left\{0,1, \ldots, \ell_{\Gamma}-1\right\}$, consider the $\mathbb{C}$-algebra $\mathscr{H}_{q}(W)$, the specialization of $\mathscr{H}_{\boldsymbol{u}}(W)$ with $u_{\Gamma, i} \mapsto q_{\Gamma, i}$. Note that replacing the collection $q_{\Gamma, i}$ with $\left(\alpha_{\Gamma} q_{\Gamma, i}\right)$ for $\alpha_{\Gamma} \in \mathbb{C}^{\times}$, we get isomorphic algebras; see, e.g., [Rouquier 2008, Section 3.3.3]. So the number of parameters actually equals $|S / W|$, where $S$ denotes the set of complex reflections in $W$. Note that if $q_{\Gamma, j}=\exp \left(2 \pi \sqrt{-1} j / \ell_{\Gamma}\right)$ for all $\Gamma$ and $j$, we just have $\mathscr{H}_{q}(W)=\mathbb{C} W$. In general, however, it is even unclear whether the algebra $\mathscr{H}_{q}(W)$ is finite-dimensional or not. In a way, the infinite dimension is the only obstruction to the equality $\operatorname{dim} \mathscr{H}_{q}(W)=|W|$. More precisely, we have the following theorem, which is the main result of this paper:

Theorem 1.1. There is a minimal two-sided ideal $I \subset \mathscr{H}_{q}(W)$ such that $\mathscr{H}_{q}(W) / I$ is finite-dimensional. Moreover, we have $\operatorname{dim} \mathscr{H}_{q}(W) / I=|W|$.

Other results towards the Broué-Malle-Rouquier conjecture were known before; see [Marin 2014] for a review. One advantage of our approach is that it is fully conceptual and does not involve any case-by-case arguments.

The key idea of the proof is to use categories 0 for rational Cherednik algebras $H_{c}(W)$, introduced in [Ginzburg et al. 2003]. By definition, the algebra $H_{c}(W)$ is the subalgebra in the skew-group algebra $D\left(\mathfrak{h}^{\text {reg }}\right) \# W$ generated by $\mathbb{C}[\mathfrak{h}], \mathbb{C} W$ and the so-called Dunkl operators $D_{a}, a \in \mathfrak{h}$. These are differential operators with firstorder poles along the reflection hyperplanes $\Gamma$. We have a triangular decomposition $H_{c}(W)=\mathbb{C}[\mathfrak{h}] \otimes \mathbb{C} W \otimes S(\mathfrak{h})$ that allows to define the category $\mathbb{O}$. This is the category of all $H_{c}(W)$-modules that are finitely generated over $\mathbb{C}[\mathfrak{h}]$ and have a locally nilpotent action of $\mathfrak{h} \subset S(\mathfrak{h})$.

Let us pick $M \in \mathfrak{O}$. Its restriction to $\mathfrak{h}^{\text {reg }}$ is a $W$-equivariant local system on $\mathfrak{h}^{\text {reg }}$. So the fiber $M_{x}$ carries a monodromy representation of $B_{W}$. It was shown in [Ginzburg et al. 2003] that the $\mathbb{C} B_{W}$-action on $M_{x}$ factors though a certain quotient of $\mathscr{H}_{q}(W)$ that has dimension $|W|$. We will show that every finite-dimensional $\mathscr{H}_{q}(W)$-module can be represented in the form $M_{x}$ for some $M \in \mathbb{O}$.

The assumption $\operatorname{dim} \mathscr{H}_{q}(W)=|W|$ is actually important for the representation theory of $H_{c}(W)$. Theorem 1.1 should make it possible to remove this assumption, but we are not going to elaborate on that.

The paper is organized as follows: In Section 2 we gather various generalities on the rational Cherednik algebras and their categories 0 . Then in Section 3 we prove the main theorem. 


\section{Generalities}

2A. Rational Cherednik algebras. Rational Cherednik algebras were introduced by Etingof and Ginzburg [2002]. In this subsection we recall their definition.

Let $W$ be a complex reflection group and $\mathfrak{h}$ be its reflection representation. We denote the subset of $W$ consisting of complex reflections by $S$. For $s \in S$, pick an eigenvector $\alpha_{s} \in \mathfrak{h}^{*}$ for $s$ with eigenvalue $\lambda_{s} \neq 1$. We fix a $W$-invariant function $c: S \rightarrow \mathbb{C}$. Using this function, for $a \in \mathfrak{h}$, we can define the Dunkl operator $D_{a} \in D\left(\mathfrak{h}^{\text {reg }}\right) \# W$ by

$$
D_{a}=\partial_{a}+\sum_{s \in S} \frac{2 c(s)}{1-\lambda_{s}} \frac{\left\langle\alpha_{s}, a\right\rangle}{\alpha_{s}}(s-1) .
$$

The rational Cherednik algebra $H_{c}(W)$ is the subalgebra in $D\left(\mathfrak{h}^{\text {reg }}\right) \# W$ generated by $\mathbb{C}[\mathfrak{h}], \mathbb{C} W$ and the Dunkl operators $D_{a}, a \in \mathfrak{h}$. Alternatively, one can present $H_{c}(W)$ by generators and relations: $H_{c}(W)$ is the quotient of $T\left(\mathfrak{h} \oplus \mathfrak{h}^{*}\right) \# W$ by the relations

$$
\left[x, x^{\prime}\right]=\left[y, y^{\prime}\right]=0,[y, x]=\langle y, x\rangle-\sum_{s \in S} c(s)\left\langle\alpha_{s}, y\right\rangle\left\langle\alpha_{s}^{\vee}, x\right\rangle s, \quad x, x^{\prime} \in \mathfrak{h}^{*}, y, y^{\prime} \in \mathfrak{h} .
$$

Here, we write $\alpha_{s}^{\vee}$ for the eigenvector of $s$ in $\mathfrak{h}$ with eigenvalue $\lambda_{s}^{-1}$ and $\left\langle\alpha_{s}^{\vee}, \alpha_{s}\right\rangle=2$. To get from the second definition to the first one, we use the homomorphism $H_{c}(W) \rightarrow D\left(\mathfrak{h}^{\text {reg }}\right) \# W$ given by $x \mapsto x, w \mapsto w, y \mapsto D_{y}$. Set $\delta:=\prod_{s \in S} \alpha_{s}^{\ell_{s}}$, where $\ell_{s}$ stands for the order of $s$ (note that this is slightly different from the usual definition). This is a $W$-invariant element, and the operator ad $\delta: H_{c}(W) \rightarrow H_{c}(W)$ is locally nilpotent, so the localization $H_{c}(W)\left[\delta^{-1}\right]$ is defined. The homomorphism $H_{c}(W) \rightarrow D\left(\mathfrak{h}^{\text {reg }}\right) \# W$ extends to an isomorphism $H_{c}(W)\left[\delta^{-1}\right] \stackrel{\sim}{\longrightarrow} D\left(\mathfrak{h}^{\text {reg }}\right) \# W$.

The algebra $H_{c}(W)$ admits a triangular decomposition: a natural map $S\left(\mathfrak{h}^{*}\right) \otimes$ $\mathbb{C} W \otimes S(\mathfrak{h}) \rightarrow H_{c}(W)$ is an isomorphism. Also $H_{c}(W)$ is graded with $\operatorname{deg} x=1$, $\operatorname{deg} w=0, \operatorname{deg} y=-1, x \in \mathfrak{h}^{*}, w \in W$, and $y \in \mathfrak{h}$. We call this grading the Euler grading. It is inner: it is given by the eigenvalues of $\operatorname{ad} h$, where

$$
h=\sum_{i=1}^{n} x_{i} y_{i}+\frac{n}{2}-\sum_{s \in S} \frac{2 c(s)}{1-\lambda_{s}} s .
$$

Now let us discuss base change for $H_{c}(W)$. Let $U$ be an affine algebraic variety equipped with an étale map $U \rightarrow \mathfrak{h} / W$. Then $\mathbb{C}[U] \otimes_{\mathbb{C}[\mathfrak{h}]^{W}} H_{c}(W)$ has a natural algebra structure; it is a subalgebra in $D\left(U \times_{\mathfrak{h} / W} \mathfrak{h}^{\text {reg }}\right) \# W$ generated by $\mathbb{C}[U] \otimes_{\mathbb{C}[\mathfrak{h} / W]} \mathbb{C}[\mathfrak{h}], \mathbb{C} W$ and the Dunkl operators. Similarly, if $U$ is a Stein complex analytic manifold (again equipped with an étale map $U \rightarrow \mathfrak{h} / W$ ), then $\mathbb{C}_{\text {an }}[U] \otimes_{\mathbb{C}[\mathfrak{h} / W]} H_{c}(W)$ is an algebra. Here and below $\mathbb{C}_{\mathrm{an}}[U]$ denotes the algebra of analytic functions on $U$. 
2B. Category 0 and $\mathbf{K Z}$ functor. The category 0 for $H_{c}(W)$ was defined in [Ginzburg et al. 2003]. By definition, it consists of all $H_{c}(W)$-modules $M$ that are finitely generated over $S\left(\mathfrak{h}^{*}\right)=\mathbb{C}[\mathfrak{h}]$ and where the action of $\mathfrak{h}$ is locally nilpotent. Equivalently, $O$ consists of all $H_{c}(W)$-modules $M$ that are finitely generated over $\mathbb{C}[\mathfrak{h}]$ and that can be graded. This category 0 will be denoted by $\mathscr{O}_{c}(W)$.

Let us proceed to the KZ functor introduced in [loc. cit., Section 5]. Pick $M \in \mathcal{O}_{c}(W)$. Then $M\left[\delta^{-1}\right]$ is a $W$-equivariant local system on $D\left(\mathfrak{h}^{\text {reg }}\right)$ with regular singularities. The category of such local systems is equivalent to the category $B_{W}$-mod $\min _{\text {fin }}$ of finite-dimensional $B_{W}$-modules; to a local system $M^{\prime}$ one assigns its fiber (or, more precisely, the fiber of its descent to $\mathfrak{h}^{\text {reg }} / W$ ) equipped with the monodromy representation. It turns out that the monodromy representation associated to $M\left[\delta^{-1}\right]$ factors through $\mathscr{H}_{q}(W)$ [loc. cit., Section 5.3], where the parameter $q$ is computed as follows: For a reflection hyperplane $\Gamma$, set

$$
\begin{aligned}
& h_{\Gamma, i}=\frac{1}{\ell_{\Gamma}} \sum_{s \in W_{\Gamma} \backslash\{1\}} \frac{2 c(s)}{\lambda_{s}-1} \lambda_{s}^{-i}, \\
& q_{\Gamma, i}=\exp \left(2 \pi \sqrt{-1}\left(h_{\Gamma, j}+j / \ell_{H}\right)\right) .
\end{aligned}
$$

So we get an exact functor $\mathrm{KZ}: \mathrm{O}_{c}(W) \rightarrow \mathscr{H}_{q}(W)-\bmod _{\text {fin }}$. This functor is given by $\operatorname{Hom}_{\mathscr{O}_{c}(W)}\left(P_{\mathrm{KZ}}, \bullet\right)$, where $P_{\mathrm{KZ}}$ is a projective object such that $\operatorname{dim}_{\operatorname{End}_{\mathscr{C}_{c}}(W)}\left(P_{\mathrm{KZ}}\right)$ is equal to $|W|$, equipped with a homomorphism $\mathscr{H}_{q}(W) \rightarrow \operatorname{End}_{\mathscr{C}_{c}}(W)\left(P_{\mathrm{KZ}}\right)^{\mathrm{opp}}$. The proof of [loc. cit., Theorem 5.15] shows that this homomorphism is surjective.

Theorem 1.1 will follow if we show that the functor KZ is essentially surjective.

2C. Isomorphisms of étale lifts. Here we are going to recall some results of [Bezrukavnikov and Etingof 2009] regarding isomorphisms of completions.

Let $W^{\prime} \subset W$ be a parabolic subgroup, i.e., the stabilizer of a point in $\mathfrak{h}$. Set $\mathfrak{h}^{\text {reg- }-W^{\prime}}:=\left\{b \in \mathfrak{h} \mid W_{b} \subset W^{\prime}\right\}$. The complement of $\mathfrak{h}^{\text {reg- }-W^{\prime}}$ in $\mathfrak{h}$ is the union of the hyperplanes $\operatorname{ker} \alpha_{s}$ for $s \notin W^{\prime}$. So $\mathfrak{h}^{\text {reg- }-W^{\prime}}$ is a principal open subset of $\mathfrak{h}$. Note that the natural morphism $\mathfrak{h}^{\text {reg- }-W^{\prime}} / W^{\prime} \rightarrow \mathfrak{h} / W$ is étale (and $\mathfrak{h}^{\text {reg- }-W^{\prime}} / W^{\prime}$ is precisely the unramified locus of $\left.\mathfrak{h} / W^{\prime} \rightarrow \mathfrak{h} / W\right)$.

Consider the space $H_{c}(W)_{\text {reg- }-W^{\prime}}:=\mathbb{C}\left[\mathfrak{h}^{\left.\text {reg- }-W^{\prime}\right]^{W^{\prime}}} \otimes_{\mathbb{C}[\mathfrak{h}]^{W}} H_{c}(W)\right.$. As was mentioned in the end of Section $2 \mathrm{~A}, H_{c}(W)_{\text {reg- }} W^{\prime}$ is actually an algebra. Bezrukavnikov and Etingof [2009, Section 3.3] essentially found an alternative description of this algebra. Namely, consider the Cherednik algebra $H_{c}\left(W^{\prime}, \mathfrak{h}\right)$ defined for the pair $W^{\prime}, \mathfrak{h}$; it decomposes into the tensor product $H_{c}\left(W^{\prime}, \mathfrak{h}\right)=D\left(\mathfrak{h}^{W^{\prime}}\right) \otimes H_{c}\left(W^{\prime}\right)$ (here we abuse notation and write $c$ for the restriction of $c$ to $S \cap W^{\prime} ; D\left(\mathfrak{h}^{W^{\prime}}\right.$ ) stands for the algebra of differential operators on $\left.\mathfrak{h}^{W^{\prime}}\right)$. Then consider its localization $H_{c}\left(W^{\prime}, \mathfrak{h}\right)_{\text {reg- }-W^{\prime}}:=\mathbb{C}\left[\mathfrak{h}^{\text {reg- }-W^{\prime}}\right]^{W^{\prime}} \otimes_{\mathbb{C}[\mathfrak{h}]^{W^{\prime}}} H_{c}\left(W^{\prime}, \mathfrak{h}\right)$. Then, following [loc. cit., Section 3.2], we can form the centralizer algebra $Z\left(W, W^{\prime}, H_{c}\left(W^{\prime}, \mathfrak{h}\right)_{\text {reg- }} W^{\prime}\right)$. Recall that, by definition, for an algebra $A$ equipped with a homomorphism $\mathbb{C} W^{\prime} \rightarrow A$, 
one defines the centralizer algebra $Z\left(W, W^{\prime}, A\right)$ as the endomorphism algebra of the right $A$-module $\operatorname{Map}_{W^{\prime}}(W, A)=\left\{f: W \rightarrow A \mid f\left(w^{\prime} w\right)=w^{\prime} f(w)\right\}$. Choosing representatives of the left $W^{\prime}$-cosets in $W$, we get an identification $Z\left(W, W^{\prime}, A\right) \cong$ $\operatorname{Mat}_{\left|W / W^{\prime}\right|}(A)$. The algebra $A$ can be recovered from $Z\left(W, W^{\prime}, A\right)$ as follows: Consider the element $e\left(W^{\prime}\right) \in Z\left(W, W^{\prime}, A\right)$ given by $e\left(W^{\prime}\right) f(u)=f(u)$ if $u \in W^{\prime}$ and 0 otherwise. Then $e\left(W^{\prime}\right) Z\left(W, W^{\prime}, A\right) e\left(W^{\prime}\right)$ is naturally identified with $A$.

The following is essentially [loc. cit., Theorem 3.2] (there, the authors considered completions instead of étale lifts, but the proof works in our situation as well).

Lemma 2.1. There is a unique isomorphism

$$
\theta: H_{c}(W)_{\mathrm{reg}-W^{\prime}} \stackrel{\sim}{\longrightarrow} Z\left(W, W^{\prime}, H_{c}\left(W^{\prime}, \mathfrak{h}\right)_{\mathrm{reg}-W^{\prime}}\right)
$$

such that the following hold for any $f \in \operatorname{Map}_{W^{\prime}}(W, A)$ and any $u \in W$ :

$$
\begin{aligned}
{[\theta(g) f](u) } & =g f(u), \quad g \in \mathbb{C}\left[\mathfrak{h}^{\mathrm{reg}-W^{\prime}}\right]^{W^{\prime}}, \\
{[\theta(\alpha) f](u) } & =(u \alpha) f(u), \quad \alpha \in \mathfrak{h}^{*}, \\
{[\theta(w) f](u) } & =f(u w), \quad w \in W, \\
{[\theta(a) f](u) } & =(u a) f(u)+\sum_{s \in S \backslash W^{\prime}} \frac{2 c(s)}{1-\lambda_{s}} \frac{\left\langle\alpha_{s}, u a\right\rangle}{\alpha_{s}}(f(s u)-f(u)), \quad a \in \mathfrak{h} .
\end{aligned}
$$

Note that the algebras in (2-4) come equipped with $\mathbb{C}^{\times}$-actions by algebra automorphisms. For example, the action of $H_{c}(W)_{\text {reg- }-W^{\prime}}$ comes from the action on $H_{c}(W)$ induced from the Euler grading and the action on $\mathfrak{h}^{\text {reg- }}-W^{\prime} / W^{\prime}$ induced from the scaling action $\left(t . x=t^{-1} x\right)$ on $\mathfrak{h}$. The isomorphism $\theta$ is $\mathbb{C}^{\times}$-equivariant.

We can further restrict $\theta$ to some analytic submanifolds or formal subschemes of $\mathfrak{h}^{\text {reg- }-W^{\prime}} / W^{\prime}$. Choose a little disk $Y \subset \mathfrak{h}^{\text {reg- }-W^{\prime}} \cap \mathfrak{h}^{W^{\prime}}$ and also a little disk $D$ around 0 in $\mathfrak{h}_{W^{\prime}} / W^{\prime}$, where $\mathfrak{h}_{W^{\prime}}$ denotes the unique $W^{\prime}$-stable complement to $\mathfrak{h}^{W^{\prime}}$ in $\mathfrak{h}$. We set $\widehat{Y}:=Y \times D$; this is an open submanifold in $\mathfrak{h}^{\text {reg }-W^{\prime}} / W^{\prime}$, in $\left(\mathfrak{h}^{\text {reg- }-W^{\prime}} \cap \mathfrak{h}^{W^{\prime}}\right) \times$ $\mathfrak{h}_{W^{\prime}} / W^{\prime}$ or in $\mathfrak{h} / W$ (under the natural morphism $\mathfrak{h}^{\text {reg }-W^{\prime}} / W^{\prime} \rightarrow \mathfrak{h} / W$ ).

So we get an isomorphism

$$
\theta_{Y}: \mathbb{C}_{\mathrm{an}}[\hat{Y}] \otimes_{\mathbb{C}[\mathfrak{h} / W]} H_{c}(W) \stackrel{\sim}{\rightarrow} Z\left(W, W^{\prime}, \mathbb{C}_{\mathrm{an}}[\hat{Y}] \otimes_{\mathbb{C}\left[\mathfrak{h} / W^{\prime}\right]} H_{c}\left(W^{\prime}, \mathfrak{h}\right)\right) .
$$

Note that this isomorphism is compatible with the Euler derivations.

We can restrict even further. Pick a point $b \in Y$, and consider the completion $\mathbb{C}[\mathfrak{h} / W]^{\wedge_{b}}$ of $\mathbb{C}[\mathfrak{h} / W]$ with respect to the maximal ideal defined by $b$. Then $\theta_{Y}$ induces

$$
\begin{aligned}
\theta_{b}: \mathbb{C}[\mathfrak{h} / W]^{\wedge_{b}} \otimes_{\mathbb{C}[\mathfrak{h} / W]} & H_{c}(W) \\
& \simeq Z\left(W, W^{\prime}, \mathbb{C}\left[\mathfrak{h} / W^{\prime}\right]^{\wedge_{b}} \otimes_{\mathbb{C}\left[\mathfrak{h} / W^{\prime}\right]} H_{c}\left(W^{\prime}, \mathfrak{h}\right)\right) .
\end{aligned}
$$

This isomorphism was originally constructed in [Bezrukavnikov and Etingof 2009]. 


\section{Proof of the main theorem}

3A. Scheme of the proof. Let $V$ be a finite-dimensional $\mathscr{H}_{q}(W)$-module and let $N$ denote the corresponding $W$-equivariant $D$-module on $\mathfrak{h}^{\text {reg }}$. Our goal is to show that there is an $M \in O_{c}(W)$ such that $M\left[\delta^{-1}\right] \cong N$. This consists of two steps:

(I) Set $\mathfrak{h}^{\text {sr }}:=\left\{b \in W \mid \operatorname{dim} \mathfrak{h}^{W_{b}} \geqslant \operatorname{dim} \mathfrak{h}-1\right\}$. This is an open subset that coincides with $\bigcup_{\Gamma \in \mathfrak{H}} \mathfrak{h}^{\text {reg- }-W_{\Gamma}}$; the codimension of its complement is bigger than 1 . We will see that there is an $O_{\mathfrak{h}^{\text {sr }}} / W^{\text {-coherent }} \mathcal{O}_{\mathfrak{h}^{\text {sr }}} / W \otimes_{\mathbb{C}[\mathfrak{h}]^{W}} H_{c}(W)$-module $\tilde{N}$ whose restriction to $\mathfrak{h}^{\text {reg }} / W$ is isomorphic to $N$ and that carries a locally finite derivation compatible with the Euler derivation of $H_{c}(W)$.

(II) We will see that $\tilde{N}$ is a vector bundle. From here we will deduce that the global sections of $\widetilde{N}$ are finitely generated and hence lie in $\mathcal{O}_{c}(W)$. Then we take $M:=\Gamma(\tilde{N})$.

Let us elaborate on how we are going to achieve (I). First, in Section 3B we will check that the Euler vector field acts on $N$ locally finitely. This will eventually prove that $\tilde{N}$ comes equipped with a locally finite derivation that is compatible with the Euler one on $H_{c}(W)$.

Now let us explain how we produce $\tilde{N}$; this is done in Section 3C. Take $W^{\prime}=W_{\Gamma}$ and let $\hat{Y}$ have the same meaning as in Section 2C. Set $\hat{Y}^{\times}:=\widehat{Y} \backslash Y$. Consider $N_{\Gamma}:=$ $e\left(W^{\prime}\right)\left(\mathbb{C}_{\mathrm{an}}\left[\hat{Y}^{\times}\right] \otimes_{\mathbb{C}\left[\mathfrak{h}^{\mathrm{reg}} / W\right]} N\right)$. This is a vector bundle on $\hat{Y}^{\times}$with a meromorphic connection that has pole of order 1 on $Y$ (since $N_{\Gamma}$ is obtained by restricting an algebraic vector bundle $e\left(W^{\prime}\right) \eta_{\Gamma}^{*} N$, where $\eta_{\Gamma}$ is a natural morphism $\mathfrak{h} / W_{\Gamma} \rightarrow \mathfrak{h} / W$, it makes sense to speak about sections of $N_{\Gamma}$ with poles on $Y$; here and below $\eta_{\Gamma}$ denotes the projection $\left.\mathfrak{h} / W_{\Gamma} \rightarrow \mathfrak{h} / W\right)$. Our first step will be to see that $N_{\Gamma}$ is obtained by restricting a $\mathbb{C}_{\mathrm{an}}[\hat{Y}] \otimes_{\mathbb{C}[\mathfrak{h} / W]} H_{c}(W)$-module $M_{\Gamma}$. Then we will see that $\left[e\left(W^{\prime}\right) \eta_{\Gamma}^{*} N\right] \cap M_{\Gamma}$ (the intersection of subspaces in $N_{\Gamma}$ ) is finitely generated over $\mathbb{C}\left[\mathfrak{h}^{\text {reg- }-W^{\prime}} / W^{\prime}\right]$. We will get $\tilde{N}$, roughly speaking, by taking the intersection of $N$ and $\eta_{\Gamma}^{*} N \cap M_{\Gamma}$ over all possible $\Gamma{ }^{1}$

3B. Locally finite derivation. Our goal here is to show that the Euler vector field acts on $N$ locally finitely. Recall that $N$ is a local system on $\mathfrak{h}^{\text {reg }} / W$ with regular singularities. Our claim is a consequence of the following general result.

Lemma 3.1. Let $X$ be the complement to a $\mathbb{C}^{\times}$-stable divisor in $\mathbb{C}^{d}$, and let $N$ be a local system with regular singularities on $X$. Then the Euler vector field eu acts on $N$ locally finitely, meaning that every $n \in N$ is included into a finite-dimensional eu-stable subspace.

${ }^{1}$ After this paper was written, I learned from Etingof that most of the proof is already contained in some form in [Wilcox 2011]. Lemmas 5.7 and 5.8 there are similar to what is done in Section 3C, while the main result of Section 3D has a somewhat easier proof in [Wilcox 2011, Lemma 3.6]. 
This claim should be standard, but we provide the proof for the sake of completeness. For a different proof, see [Wilcox 2011, Lemma 3.2].

Proof. If $N^{\prime} \subset N$ is a $D(X)$-submodule and eu acts locally finitely on $N / N^{\prime}$ and $N^{\prime}$, then the same is true for $N$. So it is enough to assume that $N$ (and hence $V$ ) is irreducible. Consider the element $\eta \in \pi_{1}(X)$ given by the loop $\exp (2 \pi \sqrt{-1} t) x_{0}$, $t \in[0,1]$, where $x_{0}$ denotes the base point. The element $\eta$ is central and hence has to act on $V$ by a scalar. Under the Riemann-Hilbert correspondence, this translates to the claim that $N$ is twisted equivariant with respect to the $\mathbb{C}^{\times}$-action. This implies our claim.

3C. Extension to codimension 1. We start by constructing $M_{\Gamma}$.

Set $\hat{Y}^{\times}:=Y \times D^{\times}=\hat{Y} \backslash Y$. Consider the category $\operatorname{Loc}_{\mathrm{rs}}(\hat{Y}, Y)$ of meromorphic local system on $\hat{Y}^{\times}$with regular singularities on $Y$ (so an object in $\operatorname{Loc}_{\mathrm{rs}}(\hat{Y}, Y)$ comes equipped with a lattice over the ring of meromorphic differential operators on $\hat{Y}^{\times}$, and a morphism in the category is supposed to preserve such lattices). The category $\operatorname{Loc}_{\mathrm{rs}}(\hat{Y}, Y)$ is equivalent to the category $\mathbb{C}\left[T^{ \pm 1}\right]$-mod of finitedimensional $\mathbb{C}\left[T^{ \pm 1}\right]$-modules via taking the monodromy representation, because $\pi_{1}\left(\hat{Y}^{\times}\right)=\pi_{1}\left(D^{\times}\right)=\mathbb{Z}$ (here we use the regular singularities condition). Under the equivalence $\mathbb{C}\left[T^{ \pm 1}\right]$-mod $\cong \operatorname{Loc}_{\mathrm{rs}}(\hat{Y}, Y)$, the $\mathrm{KZ}$ functor becomes

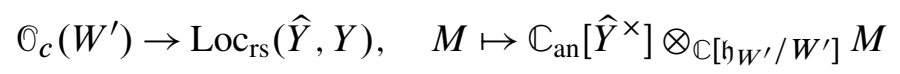

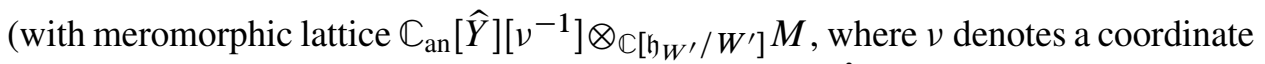
on $\left.\mathfrak{h}_{W^{\prime}} / W^{\prime}\right)$. The right adjoint $\mathrm{KZ}^{*}$ sends $N^{\prime} \in \operatorname{Loc}_{\mathrm{rs}}(\hat{Y}, Y)$ to the subspace of $N^{\prime}$ of all meromorphic elements annihilated by the vector fields on $Y$ and lying in the generalized eigenspace for $\mathfrak{h}_{W^{\prime}} \subset H_{c}\left(W^{\prime}\right)$ with eigenvalue 0 .

Now we can produce a $\mathbb{C}[\hat{Y}] \otimes_{\mathbb{C}\left[\mathfrak{h} / W^{\prime}\right]} H_{c}\left(W^{\prime}, \mathfrak{h}\right)$-module $M_{\Gamma} \in \mathcal{O}_{c}\left(W^{\prime}, \hat{Y}\right)$. Set $N_{\Gamma}=e\left(W^{\prime}\right)\left(\mathbb{C}_{\mathrm{an}}\left[\hat{Y}^{\times}\right] \otimes_{\mathbb{C}\left[\mathfrak{h}^{\mathrm{reg}} / W\right]} N\right)$. This is an object in $\operatorname{Loc}_{\mathrm{rs}}(\hat{Y}, Y)$. Note that, under the equivalence $\operatorname{Loc}_{\mathrm{rs}}(\widehat{Y}, Y) \cong \mathbb{C}\left[T^{ \pm 1}\right]$-mod, we have $N_{\Gamma} \in \mathcal{H}_{q}\left(W^{\prime}\right)$-mod. Now set

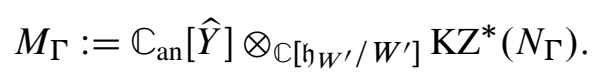

Note that the description of $\mathrm{KZ}^{*}\left(N_{\Gamma}\right) \subset N_{\Gamma}$ implies that it is stable under the Euler vector field on $N_{\Gamma}$. So $M_{\Gamma} \subset N_{\Gamma}$ is also stable under the Euler vector field.

Let $\tilde{N}_{\Gamma}:=M_{\Gamma} \cap e\left(W^{\prime}\right) \eta_{\Gamma}^{*} N$ (the intersection is taken inside $N_{\Gamma}$ ). This is a submodule in the $\mathbb{C}\left[\mathfrak{h}^{\mathrm{reg}-W^{\prime}}\right]^{W^{\prime}} \otimes_{\mathbb{C}\left[\mathfrak{h} / W^{\prime}\right]} H_{c}\left(W^{\prime}\right)$-module $e\left(W^{\prime}\right) \eta_{\Gamma}^{*} N$ which is stable under the Euler vector field.

Lemma 3.2. The module $\tilde{N}_{\Gamma}$ is finitely generated over $\mathbb{C}\left[\mathfrak{h}^{\text {reg- }-W^{\prime}}\right]^{W^{\prime}}$ and satisfies $\tilde{N}_{\Gamma}\left[v^{-1}\right]=e\left(W^{\prime}\right) \eta_{\Gamma}^{*} N$, where $v$ is a coordinate on $\mathfrak{h}_{W^{\prime}} / W^{\prime} \cong \mathbb{C}$. 
Proof. Note that the epimorphism $\mathscr{H}_{q}\left(W^{\prime}\right) \rightarrow \operatorname{End}_{\mathscr{O}_{c}\left(W^{\prime}\right)}\left(P_{\mathrm{KZ}}\right)^{\text {opp }}$ is an isomorphism. Let $N_{\Gamma}^{\text {mer }} \subset N_{\Gamma}$ denote the meromorphic lattice. Then $M_{\Gamma}$ is contained in $N_{\Gamma}^{\text {mer }}$ and is a $\mathbb{C}_{\text {an }}[\hat{Y}]$-lattice there. Indeed, it is enough to show this fiberwise (i.e., at any point of $Y$ ), where this is clear (to prove that $M_{\Gamma}$ is a lattice we use the observation that $N_{\Gamma}$ is the image of $M_{\Gamma}$ under the $\mathrm{KZ}$ functor). For any other lattice $M^{\prime}$, we have $v^{d} M^{\prime} \subset M_{\Gamma} \subset v^{-d} M^{\prime}$ for some $d>0$. So it is enough to show that $e\left(W^{\prime}\right) \eta_{\Gamma}^{*} N \cap M^{\prime}$ is finitely generated for some lattice $M^{\prime}$. Let us construct such an $M^{\prime}$.

As a $\mathbb{C}\left[\mathfrak{h}^{\text {reg }} / W\right]$-module, $N$ is projective and hence is a direct summand in a free module, say $\mathbb{C}\left[\mathfrak{h}^{\text {reg }} / W\right]^{\oplus r}$. So $N_{\Gamma}^{\text {mer }}$ is a direct summand in $\mathbb{C}_{\text {an }}[\hat{Y}]\left[v^{-1}\right]^{\oplus r}$. The intersection $M^{\prime}:=N_{\Gamma}^{\text {mer }} \cap \mathbb{C}_{\text {an }}[\widehat{Y}]^{\oplus r}$ (inside $\mathbb{C}_{\text {an }}[\hat{Y}]\left[v^{-1}\right]^{\oplus r}$ ) is clearly a lattice in $N_{\Gamma}^{\text {mer }}$. Further, the intersection $M^{\prime} \cap e\left(W^{\prime}\right) \eta_{\Gamma}^{*} N$ coincides with

$$
e\left(W^{\prime}\right) \eta_{\Gamma}^{*} N \cap \mathbb{C}\left[\mathfrak{h}^{\mathrm{reg}-W^{\prime}} / W^{\prime}\right]^{\oplus r},
$$

and hence is finitely generated (and clearly is a lattice in $\left.e\left(W^{\prime}\right) \eta_{\Gamma}^{*} N\right)$.

Now we are ready to define a module $\tilde{N}$ over $\mathcal{O}_{\mathfrak{h}}$ sr $/ W \otimes_{\mathbb{C}[\mathfrak{h} / W]} H_{c}(W)$. Abusing notation, we will write $\tilde{N}_{\Gamma}$ for the corresponding (under the equivalence) $\mathbb{C}\left[\mathfrak{h}^{\text {reg- }} W_{\Gamma} / W_{\Gamma}\right] \otimes_{\mathbb{C}[\mathfrak{h} / W]} H_{c}(W)$-module. The restriction of $\tilde{N}_{\Gamma}$ to $\eta_{\Gamma}^{-1}\left(\mathfrak{h}^{\text {reg }} / W\right)$ coincides with $\eta_{\Gamma}^{*} N$ by construction. Let $\iota_{\Gamma}: \mathfrak{h}^{\text {reg- }-W_{\Gamma}} \hookrightarrow \mathfrak{h}$ be the inclusion and $\pi_{\Gamma}: \mathfrak{h}^{\text {reg- }-W_{\Gamma}} \rightarrow \mathfrak{h}^{\text {reg- }-W_{\Gamma}} / W_{\Gamma}$ be the quotient morphism. Also let $\pi: \mathfrak{h} \rightarrow \mathfrak{h} / W$ denote the quotient morphism and $\iota: \mathfrak{h}^{\text {reg }} \hookrightarrow \mathfrak{h}^{\text {sr }}$ the inclusion. Note that, by the construction, $\iota_{\Gamma *} \pi_{\Gamma}^{*} \tilde{N}_{\Gamma} \subset \iota_{*} \pi^{*} N$ (recall that we view $N$ as a coherent sheaf on $\left.\mathfrak{h}^{\text {reg }} / W\right)$. The intersection $\widehat{N}:=\bigcap_{\Gamma}{ }^{\iota} \Gamma * \pi_{\Gamma}^{*} \tilde{N}_{\Gamma}$ is a coherent sheaf on $\mathfrak{h}^{\text {sr }}$ because of Lemma 3.2 and the equality $\mathfrak{h}^{\text {sr }}=\bigcup_{\Gamma} \mathfrak{h}^{\text {reg- }} W_{\Gamma}$. The intersection is stable under the Euler vector field because all the $\widetilde{N}_{\Gamma}$ are. Also $\hat{N}$ is $W$-stable; this is because $w \pi_{\Gamma}^{*} \tilde{N}_{\Gamma}=\pi_{w \Gamma}^{*} \tilde{N}_{w \Gamma}$. Now set $\tilde{N}:=\pi_{*}(\hat{N})^{W}=\pi_{*} \hat{N} \cap \iota_{*}^{\prime} N$ (where $\iota^{\prime}: \mathfrak{h}^{\text {sr }} / W \hookrightarrow \mathfrak{h} / W$ denotes the inclusion). This is a coherent sheaf on $\mathfrak{h}^{\text {sr }} / W$, stable under the Euler vector field on $\iota_{*}^{\prime} N$. It remains to show that $\tilde{N} \subset \iota_{*}^{\prime} N$ is stable under $H_{c}(W)$. But this follows from the equality

$$
\tilde{N}=\iota_{*}^{\prime} N \cap \bigcap_{\Gamma} \eta_{\Gamma *} \tilde{N}_{\Gamma},
$$

where now we view $\eta_{\Gamma}$ as a morphism $\mathfrak{h}^{\text {reg- }-W^{\prime}} / W^{\prime} \rightarrow \mathfrak{h}^{\text {sr }} / W$. Equation (3-2) follows from the observation that $\tilde{N}_{\Gamma}=\pi_{\Gamma *}\left(\pi_{\Gamma}^{*} \widetilde{N}_{\Gamma}\right)^{W_{\Gamma}}$ and $\pi=\eta_{\Gamma} \circ \pi_{\Gamma}$. Since all sheaves in the right-hand side of (3-2) are stable under $H_{c}(W)$, we see that $\tilde{N}$ is stable as well. It follows from the construction that $\left.\tilde{N}\right|_{\mathfrak{h}^{\text {reg }} / W} \cong N$.

\section{D. Global sections.}

Lemma 3.3. The sheaf $\tilde{N}$ is a vector bundle on $\mathfrak{h}^{\text {sr }} / W$. 
Proof. The proof is inspired by [Etingof et al. 2013, Section 3.2]. We need to show that $\tilde{N}$ is maximal Cohen-Macaulay when viewed as a coherent sheaf on $\mathfrak{h}^{\text {sr }} / W$. Let $Z$ denote the non-CM locus of $\tilde{N}$ in $\mathfrak{h}^{\text {sr }} / W$ and let $d$ be the codimension of $Z$ in $\mathfrak{h}^{\text {sr }} / W$. Pick an open $\mathbb{C}^{\times}$-stable affine subvariety $U$ of $\mathfrak{h}^{\text {sr }} / W$ that intersects $Z$ (or, more precisely, an irreducible component of maximal dimension in $Z$ ). Consider $H_{U \cap Z}^{i}(U, \widetilde{N})$ for $i<d$. As in [Etingof et al. 2013, Section 3.2], all these groups are $\mathbb{C}[U] \otimes_{\mathbb{C}[\mathfrak{h} / W]} H_{c}(W)$-modules, finitely generated over $\mathbb{C}[U]$ (this follows from [SGA 2 1968, Exposé VIII, Corollary 2.3] using equivalence of (ii) and (iii) there; note that the depth used in (ii) coincides with the codimension thanks to the choice of $Z$ ). Moreover, by the choice of $Z$, one of these modules is nonzero, as in [Etingof et al. 2013, Section 3.2]. The support of $R:=H_{U \cap Z}^{i}(U, \tilde{N})$ is contained in $Z \cap U$.

Pick $b \in \mathfrak{h}$ lying over the support of $R$. Recall the isomorphism $\theta_{b}: H_{c}(W)^{\wedge_{b}} \cong$ $Z\left(W, W^{\prime}, H_{c}\left(W^{\prime}, \mathfrak{h}\right)^{\wedge_{b}}\right)$, where we take $W^{\prime}$ to be $W_{b}$. So we get a nonzero $H_{c}\left(W^{\prime}, \mathfrak{h}\right)^{\wedge_{b}}$-module $e\left(W^{\prime}\right) \theta_{b *}\left(R^{\wedge_{b}}\right)$. This module is finitely generated over $\mathbb{C}[\mathfrak{h}]^{\wedge_{b}}$. So it is of the form $\mathbb{C}\left[\mathfrak{h}_{W^{\prime}}\right]^{\wedge_{b}} \otimes R_{0}^{\wedge_{0}}$ for $R_{0} \in \mathscr{O}_{c}\left(W^{\prime}\right)$. It follows that $d=1$ and that $R=\Gamma_{Z \cap U}(U, \tilde{N})$. But, by construction, $\Gamma(U, \tilde{N})$ is embedded into $\Gamma\left(U \cap \mathfrak{h}^{\text {reg }} / W, N\right)$ and so $\Gamma(U, \widetilde{N})$ has no torsion $\mathbb{C}[U]$-submodules. We get a contradiction, showing that $\tilde{N}$ is Cohen-Macaulay. Since $\tilde{N}$ is torsion-free, we see that it is maximal Cohen-Macaulay, and hence is a vector bundle.

Now we can use [SGA 2 1968, Exposé VIII, Corollary 2.3(iv)] (applied to an extension of $\tilde{N}$ to a coherent sheaf on $\mathfrak{h} / W)$ to see that $M:=\Gamma\left(\mathfrak{h}^{\text {sr }} / W, \tilde{N}\right)$ is finitely generated over $\mathbb{C}[\mathfrak{h}]^{W}$. Let us show that the $H_{c}(W)$-module $M$ lies in $\mathrm{O}_{c}(W)$. By construction, $M$ carries a locally finite derivation compatible with the derivation ad $h$ of $H_{c}(W)$. It follows that $M$ is gradable and hence lies in 0 . Also, by construction, $M\left[\delta^{-1}\right]=N$. This completes the proof.

\section{Acknowledgements}

This project was sparked by a discussion with Raphael Rouquier in April 2014. I would like to thank him and Roman Bezrukavnikov, Pavel Etingof and Dennis Gaitsgory for stimulating discussions. Furthermore, I am grateful to Pavel Etingof for his comments on a preliminary version of this paper and for pointing out the reference [Wilcox 2011], and Daniel Thompson for his careful reading of the paper. My work was supported by the NSF under grant DMS-1161584.

\section{References}

[Bezrukavnikov and Etingof 2009] R. Bezrukavnikov and P. Etingof, "Parabolic induction and restriction functors for rational Cherednik algebras", Selecta Math. (N.S.) 14:3-4 (2009), 397-425. MR 2010e:20007 Zbl 1226.20002 
[Broué et al. 1998] M. Broué, G. Malle, and R. Rouquier, "Complex reflection groups, braid groups, Hecke algebras”, J. Reine Angew. Math. 500 (1998), 127-190. MR 99m:20088 Zbl 0921.20046

[Etingof and Ginzburg 2002] P. Etingof and V. Ginzburg, "Symplectic reflection algebras, CalogeroMoser space, and deformed Harish-Chandra homomorphism", Invent. Math. 147:2 (2002), 243-348. MR 2003b:16021 Zbl 1061.16032

[Etingof et al. 2013] P. Etingof, E. Gorsky, and I. Losev, "Representations of rational Cherednik algebras with minimal support and torus knots", preprint, 2013. arXiv 1304.3412

[Ginzburg et al. 2003] V. Ginzburg, N. Guay, E. Opdam, and R. Rouquier, "On the category 0 for rational Cherednik algebras", Invent. Math. 154:3 (2003), 617-651. MR 2005f:20010 Zbl 1071.20005

[Marin 2014] I. Marin, "The freeness conjecture for Hecke algebras of complex reflection groups, and the case of the Hessian group $G_{26}$ ", J. Pure Appl. Algebra 218:4 (2014), 704-720. MR 3133700 Zbl 1291.20042

[Rouquier 2008] R. Rouquier, “ $q$-Schur algebras and complex reflection groups", Mosc. Math. J. 8:1 (2008), 119-158. MR 2010b:20081 Zbl 1213.20007

[SGA 2 1968] A. Grothendieck, Cohomologie locale des faisceaux cohérents et théorèmes de Lefschetz locaux et globaux (Séminaire de Géométrie Algébrique du Bois Marie $1962=$ SGA 2), Advanced Stud. in Pure Math. 2, North-Holland, Amsterdam, 1968. MR 57 \#16294 Zbl 0197.47202

[Shephard and Todd 1954] G. C. Shephard and J. A. Todd, "Finite unitary reflection groups", Canadian J. Math. 6 (1954), 274-304. MR 15,600b Zbl 0055.14305

[Wilcox 2011] S. Wilcox, "Supports of representations of the rational Cherednik algebra of type A", preprint, 2011. arXiv 1012.2585

Communicated by J. Toby Stafford

Received 2014-08-13 Accepted 2015-02-18

i.loseu@neu.edu

Department of Mathematics, Northeastern University, Boston, MA 02115, United States 


\section{Algebra \& Number Theory}

msp.org/ant

\section{EDITORS}

MANAGING EDITOR

Bjorn Poonen

Massachusetts Institute of Technology

Cambridge, USA

\author{
EDITORIAL BOARD CHAIR \\ David Eisenbud \\ University of California \\ Berkeley, USA
}

BOARD OF EDITORS

Georgia Benkart

Dave Benson

Richard E. Borcherds

John H. Coates

J-L. Colliot-Thélène

Brian D. Conrad

Hélène Esnault

Hubert Flenner

Edward Frenkel

Andrew Granville

Joseph Gubeladze

Roger Heath-Brown

Craig Huneke

János Kollár

Yuri Manin

Barry Mazur

Philippe Michel

Susan Montgomery
University of Wisconsin, Madison, USA

University of Aberdeen, Scotland

University of California, Berkeley, USA

University of Cambridge, UK

CNRS, Université Paris-Sud, France

University of Michigan, USA

Freie Universität Berlin, Germany

Ruhr-Universität, Germany

University of California, Berkeley, USA

Université de Montréal, Canada

San Francisco State University, USA

Oxford University, UK

University of Virginia, USA

Princeton University, USA

Northwestern University, USA

Harvard University, USA

École Polytechnique Fédérale de Lausanne

University of Southern California, USA
Shigefumi Mori

Raman Parimala

Jonathan Pila

Anand Pillay

Victor Reiner

Peter Sarnak

Joseph H. Silverman

Michael Singer

Vasudevan Srinivas

J. Toby Stafford

Richard Taylor

Ravi Vakil

Michel van den Bergh

Marie-France Vignéras

Kei-Ichi Watanabe

Efim Zelmanov

Shou-Wu Zhang
RIMS, Kyoto University, Japan

Emory University, USA

University of Oxford, UK

University of Notre Dame, USA

University of Minnesota, USA

Princeton University, USA

Brown University, USA

North Carolina State University, USA

Tata Inst. of Fund. Research, India

University of Michigan, USA

Harvard University, USA

Stanford University, USA

Hasselt University, Belgium

Université Paris VII, France

Nihon University, Japan

University of California, San Diego, USA

Princeton University, USA

\section{PRODUCTION}

production@msp.org

Silvio Levy, Scientific Editor

See inside back cover or msp.org/ant for submission instructions.

The subscription price for 2015 is US \$255/year for the electronic version, and $\$ 440 /$ year ( $\$ 55$, if shipping outside the US) for print and electronic. Subscriptions, requests for back issues and changes of subscribers address should be sent to MSP.

Algebra \& Number Theory (ISSN 1944-7833 electronic, 1937-0652 printed) at Mathematical Sciences Publishers, 798 Evans Hall \#3840, c/o University of California, Berkeley, CA 94720-3840 is published continuously online. Periodical rate postage paid at Berkeley, CA 94704, and additional mailing offices.

ANT peer review and production are managed by EditFLOW ${ }^{\circledR}$ from MSP.

\section{PUBLISHED BY}

7 mathematical sciences publishers

nonprofit scientific publishing

http://msp.org/

(C) 2015 Mathematical Sciences Publishers 


\section{Algebra \& Number Theory}

Volume $9 \quad$ No. $2 \quad 2015$

Lifting harmonic morphisms II: Tropical curves and metrized complexes

OMID AMINI, MATTHEW BAKER, ERWAN BRUgallé and JosePH RABINOFF

Noncommutative Hilbert modular symbols

IVAN HOROZOV

$p$-adic Hodge theory in rigid analytic families

REBECCA BELLOVIN

Semistable periods of finite slope families

RUOCHUAN LIU

ANAND DEOPURKAR and ANAND PATEL

Finite-dimensional quotients of Hecke algebras

493

IVAN LOSEV

Semiample invertible sheaves with semipositive continuous hermitian metrics

503 ATSUSHI MORIWAKI 